Analele Universităţii de Vest, Timişoara

Seria Matematică - Informatică

LIII, 1, (2015), 163- 175

\title{
Random fixed point theorems for Ciric quasi contraction in cone random metric spaces
}

G. S. Saluja

\begin{abstract}
The purpose of this paper is to establish a common random fixed point theorem by using Ciric quasi contraction for two random operators in the framework of cone random metric spaces and also to obtain some random fixed point results as corollaries. Our results extend and generalize the corresponding recent result from the current existing literature.
\end{abstract}

AMS Subject Classification (2000). 47H10, 54H25.

Keywords. Cone random metric space, common random fixed point, Ciric quasi contraction, cone.

\section{Introduction}

Random nonlinear analysis is an important mathematical discipline which is mainly concerned with the study of random nonlinear operators and their properties and is needed for the study of various classes of random equations. The study of random fixed point theory was initiated by the Prague school of Probabilities in the 1950s $[12,13,28]$. Common random fixed point theorems are stochastic generalization of classical common fixed point theorems. The machinery of random fixed point theory provides a convenient way of modeling many problems arising from economic theory (see e.g. [22]) and references mentioned therein. Random methods have revolutionized the financial markets. The survey article by Bharucha-Reid [9] attracted the attention 
of several mathematicians and gave wings to the theory. Itoh [17] extended Spacek's and Hans's theorem to multivalued contraction mappings (see, also [2], [3], [23], [24]). Now this theory has become the full fledged research area and various ideas associated with random fixed point theory are used to obtain the solution of nonlinear random system (see $[6-8,14,26]$ ). Papageorgiou [20,21], Beg [4,5] studied common random fixed points and random coincidence points of a pair of compatible random operators and proved fixed point theorems for contractive random operators in Polish spaces.

In 2007, Huang and Zhang [15] introduced the concept of cone metric spaces and establish some fixed point theorems for contractive mappings in normal cone metric spaces. Subsequently, several other authors $[1,16,25,27]$ studied the existence of fixed points and common fixed points of mappings satisfying contractive type condition on a normal cone metric space.

In 2008, Rezapour and Hamlbarani [25] omitted the assumption of normality in cone metric space, which is a milestone in developing fixed point theory in cone metric space. Recently, Mehta et al. [19] introduced the concept of cone random metric space and proved the existence of random fixed point under weak contraction condition in the setting of cone random metric spaces.

\section{Preliminaries}

Definition 2.1. (See [19]) Let $(E, \tau)$ be a topological vector space. A subset $P$ of $E$ is called a cone whenever the following conditions hold:

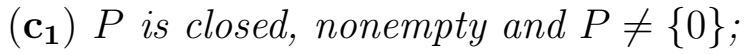

$\left(\mathbf{c}_{2}\right) a, b \in R, a, b \geq 0$ and $x, y \in P$ imply $a x+b y \in P$;

(c) If $x \in P$ and $-x \in P$ implies $x=0$.

For a given cone $P \subset E$, we define a partial ordering $\leq$ with respect to $P$ by $x \leq y$ if and only if $y-x \in P$. We shall write $x<y$ to indicate that $x \leq y$ but $x \neq y$, while $x \ll y$ will stand for $y-x \in P^{0}$, where $P^{0}$ stands for the interior of $P$.

Definition 2.2. (See $[15,29]$ ) Let $X$ be a nonempty set. Suppose that the mapping $d: X \times X \rightarrow E$ satisfies: 
Vol. LIII (2015) Random fixed point theorems for Ciric quasi ......

$\left(\mathbf{d}_{\mathbf{1}}\right) 0 \leq d(x, y)$ for all $x, y \in X$ and $d(x, y)=0$ if and only if $x=y$;

$\left(\mathbf{d}_{\mathbf{2}}\right) d(x, y)=d(y, x)$ for all $x, y \in X$

$\left(\mathbf{d}_{\mathbf{3}}\right) d(x, y) \leq d(x, z)+d(z, y) x, y, z \in X$.

Then $d$ is called a cone metric [15] or K-metric [29] on $X$ and $(X, d)$ is called a cone metric space [15].

The concept of a cone metric space is more general than that of a metric space, because each metric space is a cone metric space where $E=\mathbb{R}$ and $P=[0,+\infty)$.

Definition 2.3. (See [15]) Let $(X, d)$ be a cone metric space. We say that $\left\{x_{n}\right\}$ is:

(i) a Cauchy sequence if for every $\varepsilon$ in $E$ with $0 \ll \varepsilon$, then there is an $N$ such that for all $n, m>N, d\left(x_{n}, x_{m}\right) \ll \varepsilon$;

(ii) a convergent sequence if for every $\varepsilon$ in $E$ with $0 \ll \varepsilon$, then there is an $N$ such that for all $n>N, d\left(x_{n}, x\right) \ll \varepsilon$ for some fixed $x$ in $X$.

A cone metric space $X$ is said to be complete if every Cauchy sequence in $X$ is convergent in $X$.

In the following $(X, d)$ will stands for a cone metric space with respect to a cone $P$ with $P^{0} \neq \emptyset$ in a real Banach space $E$ and $\leq$ is partial ordering in $E$ with respect to $P$.

Definition 2.4. (Measurable function) (See [19]) Let $(\Omega, \Sigma)$ be a measurable space with $\Sigma$-a sigma algebra of subsets of $\Omega$ and $M$ be a nonempty subset of a metric space $X=(X, d)$. Let $2^{M}$ be the family of nonempty subsets of $M$ and $C(M)$ the family of all nonempty closed subsets of $M$. A mapping $G: \Omega \rightarrow 2^{M}$ is called measurable if for each open subset $U$ of $M$, $G^{-1}(U) \in \Sigma$, where $G^{-1}(U)=\{\omega \in \Omega: G(\omega) \cap U \neq \emptyset\}$.

Definition 2.5. (Measurable selector) (See [19]) A mapping $\xi: \Omega \rightarrow M$ is called a measurable selector of a measurable mapping $G: \Omega \rightarrow 2^{M}$ if $\xi$ is measurable and $\xi(\omega) \in G(\omega)$ for each $\omega \in \Omega$.

Definition 2.6. (Random operator) (See [19]) The mapping $T: \Omega \times M \rightarrow$ $X$ is said to be a random operator if and only if for each fixed $x \in M$, the mapping $T(., x): \Omega \rightarrow X$ is measurable. 
Definition 2.7. (Continuous random operator) (See [19]) A random operator $T: \Omega \times M \rightarrow X$ is said to be continuous random operator if for each fixed $x \in M$ and $\omega \in \Omega$, the mapping $T(\omega,):. X \rightarrow X$ is continuous.

Definition 2.8. (Random fixed point) (See [19]) A measurable mapping $\xi: \Omega \rightarrow M$ is a random fixed point of a random operator $T: \Omega \times M \rightarrow X$ if and only if $T(\omega, \xi(\omega))=\xi(\omega)$ for each $\omega \in \Omega$.

Definition 2.9. (Cone Random Metric Space) (See [19]) Let $M$ be a nonempty set and the mapping $d: \Omega \times M \rightarrow P$, where $P$ is a cone, $\omega \in \Omega$ be a selector, satisfy the following conditions:

(i) $d(x(\omega), y(\omega)) \geq 0$ and $d(x(\omega), y(\omega))=0$ if and only if $x(\omega)=y(\omega)$ for all $(x(\omega), y(\omega)) \in \Omega \times M$;

(ii) $d(x(\omega), y(\omega))=d(y(\omega), x(\omega))$ for all $x, y \in M, \omega \in \Omega$ and $x(\omega), y(\omega)$ $\in \Omega \times M$

(iii) $d(x(\omega), y(\omega)) \leq d(x(\omega), z(\omega))+d(z(\omega), y(\omega))$ for all $x, y, z \in M$ and $\omega \in \Omega$ a selector;

(iv) for any $x, y \in M, \omega \in \Omega, d(x(\omega), y(\omega))$ is non-increasing and left continuous.

Then $d$ is called a cone random metric on $M$ and $(M, d)$ is called a cone random metric space.

Definition 2.10. Let $(X, d)$ be a metric space. A mapping $T: X \rightarrow X$ is called an a-contraction if

$$
d(T x, T y) \leq a d(x, y) \text { for all } x, y \in X,
$$

where $a \in(0,1)$.

Definition 2.11. The mapping $T$ is called Kannan contraction mapping [18] if there exists $b \in\left(0, \frac{1}{2}\right)$ such that

$$
d(T x, T y) \leq b[d(x, T x)+d(y, T y)] \text { for all } x, y \in X .
$$

Definition 2.12. The mapping $T$ is called Chatterjea contraction mapping [11] if there exists $c \in\left(0, \frac{1}{2}\right)$ such that

$$
d(T x, T y) \leq c[d(x, T y)+d(y, T x)] \text { for all } x, y \in X .
$$


Combining these three definitions, Zamfirescu [30] proved the following important result.

Theorem Z . Let $(X, d)$ be a complete metric space and $T: X \rightarrow X$ a mapping for which there exists the real number $a, b$ and $c$ satisfying $a \in(0,1), b, c \in\left(0, \frac{1}{2}\right)$ such that for any pair $x, y \in X$, at least one of the following conditions holds:

$$
\begin{aligned}
& \left(z_{1}\right) d(T x, T y) \leq a d(x, y), \\
& \left(z_{2}\right) d(T x, T y) \leq b[d(x, T x)+d(y, T y)], \\
& \left(z_{3}\right) d(T x, T y) \leq c[d(x, T y)+d(y, T x)] .
\end{aligned}
$$

Then $T$ has a unique fixed point $p$ and the Picard iteration $\left\{x_{n}\right\}_{n=0}^{\infty}$ defined by $x_{n+1}=T x_{n}, n=0,1,2, \ldots$ converges to $p$ for any arbitrary but fixed $x_{0} \in X$.

An operator $T$ which satisfies at least one of the contractive conditions $\left(z_{1}\right),\left(z_{2}\right)$ and $\left(z_{3}\right)$ is called a Zamfirescu operator or a $Z$-operator.

The conditions $\left(z_{1}\right)-\left(z_{3}\right)$ can be written in the following equivalent form

$$
\begin{aligned}
& d(T x, T y) \leq h \max \left\{d(x, y), \frac{d(x, T x)+d(y, T y)}{2},\right. \\
& \left.\frac{d(x, T y)+d(y, T x)}{2}\right\} \text {, }
\end{aligned}
$$

for all $x, y \in X ; 0<h<1$, has been obtained by Ciric [10] in 1974 .

A mapping satisfying (2.4) is commonly called Ciric quasi contraction. It is obvious that each of the conditions $\left(z_{1}\right)-\left(z_{3}\right)$ implies $(2.4)$.

The purpose of this paper is to establish a common random fixed point theorem for two random operators by using condition (2.4) in the framework of cone random metric spaces and also to obtain some random fixed point results as corollaries. 


\section{Main Results}

In this section we shall prove common random fixed point theorems under contractive condition (2.4) in the framework of cone random metric spaces.

Theorem 3.1. Let $(X, d)$ be a complete cone random metric space with respect to a cone $P$ and let $M$ be a nonempty separable closed subset of $X$. Let $S$ and $T$ be two continuous random operators defined on $M$ such that for $\omega \in \Omega, S(\omega,),. T(\omega,):. \Omega \times M \rightarrow M$ satisfying the condition

$$
\begin{aligned}
d(S(x(\omega)), T(y(\omega))) \leq & h \max \{d(x(\omega), y(\omega)) \\
& \frac{d(x(\omega), S(x(\omega)))+d(y(\omega), T(y(\omega)))}{2} \\
& \left.\frac{d(x(\omega), T(y(\omega)))+d(y(\omega), S(x(\omega)))}{2}\right\},
\end{aligned}
$$

for all $x, y \in X ; 0<h<1$ and $\omega \in \Omega$. Then $S$ and $T$ have a unique common random fixed point in $X$.

Proof. For each $x_{0}(\omega) \in \Omega \times X$ and $n=0,1,2, \ldots$, we choose $x_{1}(\omega), x_{2}(\omega)$ $\in \Omega \times X$ such that $x_{1}(\omega)=S\left(x_{0}(\omega)\right)$ and $x_{2}(\omega)=T\left(x_{1}(\omega)\right)$. In general we define sequence of elements of $X$ such that $x_{2 n+1}(\omega)=S\left(x_{2 n}(\omega)\right)$ and $x_{2 n+2}(\omega)=T\left(x_{2 n+1}(\omega)\right)$. Then from (3.1), we have

$$
\begin{aligned}
d\left(x_{2 n+1}(\omega), x_{2 n}(\omega)\right)= & d\left(S\left(x_{2 n}(\omega)\right), T\left(x_{2 n-1}(\omega)\right)\right) \\
\leq & h \max \left\{d\left(x_{2 n}(\omega), x_{2 n-1}(\omega)\right),\right. \\
& \frac{d\left(x_{2 n}(\omega), S\left(x_{2 n}(\omega)\right)\right)+d\left(x_{2 n-1}(\omega), T\left(x_{2 n-1}(\omega)\right)\right)}{2}, \\
& \left.\frac{d\left(x_{2 n}(\omega), T\left(x_{2 n-1}(\omega)\right)\right)+d\left(x_{2 n-1}(\omega), S\left(x_{2 n}(\omega)\right)\right)}{2}\right\} \\
= & h \max \left\{d\left(x_{2 n}(\omega), x_{2 n-1}(\omega)\right),\right. \\
& \frac{d\left(x_{2 n}(\omega), x_{2 n+1}(\omega)\right)+d\left(x_{2 n-1}(\omega), x_{2 n}(\omega)\right)}{2}, \\
& \left.\frac{d\left(x_{2 n}(\omega), x_{2 n}(\omega)\right)+d\left(x_{2 n-1}(\omega), x_{2 n+1}(\omega)\right)}{2}\right\}
\end{aligned}
$$


Vol. LIII (2015) Random fixed point theorems for Ciric quasi ......

$$
\begin{aligned}
= & h \max \left\{d\left(x_{2 n}(\omega), x_{2 n-1}(\omega)\right),\right. \\
& \frac{d\left(x_{2 n}(\omega), x_{2 n+1}(\omega)\right)+d\left(x_{2 n-1}(\omega), x_{2 n}(\omega)\right)}{2}, \\
& \left.\frac{d\left(x_{2 n-1}(\omega), x_{2 n+1}(\omega)\right)}{2}\right\} \\
= & h \max \left\{d\left(x_{2 n}(\omega), x_{2 n-1}(\omega)\right),\right. \\
& \frac{d\left(x_{2 n}(\omega), x_{2 n+1}(\omega)\right)+d\left(x_{2 n-1}(\omega), x_{2 n}(\omega)\right)}{2}, \\
& \left.\frac{d\left(x_{2 n-1}(\omega), x_{2 n}(\omega)\right)+d\left(x_{2 n}(\omega), x_{2 n+1}(\omega)\right)}{2}\right\} .
\end{aligned}
$$

Since for non-negative real numbers $a$ and $b$, we have

$$
\frac{a+b}{2} \leq \max \{a, b\}
$$

Therefore, we have

$$
\begin{aligned}
\frac{d\left(x_{2 n}(\omega), x_{2 n+1}(\omega)\right)+d\left(x_{2 n-1}(\omega), x_{2 n}(\omega)\right)}{2} \leq & \max \left\{d\left(x_{2 n+1}(\omega), x_{2 n}(\omega)\right),\right. \\
& \left.d\left(x_{2 n}(\omega), x_{2 n-1}(\omega)\right)\right\} .
\end{aligned}
$$

Using (3.4) in (3.2), we have

$$
\begin{aligned}
d\left(x_{2 n+1}(\omega), x_{2 n}(\omega)\right) \leq & h \max \left\{d\left(x_{2 n}(\omega), x_{2 n-1}(\omega)\right)\right. \\
& d\left(x_{2 n+1}(\omega), x_{2 n}(\omega)\right), d\left(x_{2 n}(\omega), x_{2 n-1}(\omega)\right) \\
& \left.d\left(x_{2 n+1}(\omega), x_{2 n}(\omega)\right), d\left(x_{2 n}(\omega), x_{2 n-1}(\omega)\right)\right\} \\
\leq & h d\left(x_{2 n}(\omega), x_{2 n-1}(\omega)\right) .
\end{aligned}
$$

Similarly, we have

$$
d\left(x_{2 n}(\omega), x_{2 n-1}(\omega)\right) \leq h d\left(x_{2 n-1}(\omega), x_{2 n-2}(\omega)\right) .
$$

Hence

$$
d\left(x_{2 n+1}(\omega), x_{2 n}(\omega)\right) \leq h^{2} d\left(x_{2 n-1}(\omega), x_{2 n-2}(\omega)\right) .
$$

On continuing this process, we get

$$
d\left(x_{2 n+1}(\omega), x_{2 n}(\omega)\right) \leq h^{2 n} d\left(x_{1}(\omega), x_{0}(\omega)\right) .
$$


Also for $n>m$, we have

$$
\begin{aligned}
d\left(x_{n}(\omega), x_{m}(\omega)\right) \leq & d\left(x_{n}(\omega), x_{n-1}(\omega)\right)+d\left(x_{n-1}(\omega), x_{n-2}(\omega)\right)+\ldots \\
& +d\left(x_{m+1}(\omega), x_{m}(\omega)\right) \\
\leq & \left(h^{n-1}+h^{n-2}+\cdots+h^{m}\right) d\left(x_{1}(\omega), x_{0}(\omega)\right) \\
\leq & \left(\frac{h^{m}}{1-h}\right) d\left(x_{1}(\omega), x_{0}(\omega)\right) .
\end{aligned}
$$

Let $0 \ll \varepsilon$ be given. Choose a natural number $N$ such that $\left(\frac{h^{m}}{1-h}\right) d\left(x_{1}(\omega), x_{0}(\omega)\right) \ll \varepsilon$ for every $m \geq N$. Thus

$$
d\left(x_{n}(\omega), x_{m}(\omega)\right) \leq\left(\frac{h^{m}}{1-h}\right) d\left(x_{1}(\omega), x_{0}(\omega)\right) \ll \varepsilon,
$$

for every $n>m \geq N$.

This shows that the sequence $\left\{x_{n}(\omega)\right\}$ is a Cauchy sequence in $\Omega \times X$. Since $(X, d)$ is complete, there exists $z(\omega) \in \Omega \times X$ such that $x_{n}(\omega) \rightarrow z(\omega)$ as $n \rightarrow \infty$. Hence, we have

$$
\begin{aligned}
d(z(\omega), S(z(\omega))) \leq & d\left(z(\omega), x_{2 n+2}(\omega)\right)+d\left(x_{2 n+2}(\omega), S(z(\omega))\right. \\
= & d\left(z(\omega), x_{2 n+2}(\omega)\right)+d\left(S \left(z(\omega), T\left(x_{2 n+1}(\omega)\right)\right.\right. \\
\leq & d\left(z(\omega), x_{2 n+2}(\omega)\right) \\
& +h \max \left\{d\left(z(\omega), x_{2 n+1}(\omega)\right),\right. \\
& \frac{d(z(\omega), S(z(\omega)))+d\left(x_{2 n+1}(\omega), T\left(x_{2 n+1}(\omega)\right)\right)}{2}, \\
& \left.\frac{d\left(z(\omega), T\left(x_{2 n+1}(\omega)\right)\right)+d\left(x_{2 n+1}(\omega), S(z(\omega))\right)}{2}\right\} \\
=\quad \begin{array}{l}
d\left(z(\omega), x_{2 n+2}(\omega)\right) \\
+
\end{array} & \frac{d(z(\omega), S(z(\omega)))+d\left(x_{2 n+1}(\omega), x_{2 n+2}(\omega)\right)}{2}, \\
& \left.\frac{d\left(z(\omega), x_{2 n+2}(\omega)\right)+d\left(x_{2 n+1}(\omega), S(z(\omega))\right)}{2}\right\} .
\end{aligned}
$$

Taking the limit as $n \rightarrow \infty$ in the above inequality, we get

$$
\begin{aligned}
d(z(\omega), S(z(\omega))) & \leq \frac{h}{2} d(z(\omega), S(z(\omega))) \\
& \leq h d(z(\omega), S(z(\omega)))
\end{aligned}
$$


or,

$(1-h) d(z(\omega), S(z(\omega))) \leq 0 \Rightarrow d(z(\omega), S(z(\omega))) \leq 0$, since $0<1-h<1$.

Thus $-d(z(\omega), S(z(\omega))) \in P$. But $d(z(\omega), S(z(\omega))) \in P$. Therefore by Definition 2.1(c $\left.c_{3}\right)$, we have $d(z(\omega), S(z(\omega))=0$ and so $S(z(\omega))=z(\omega)$.

In an exactly similar way we can prove that for all $\omega \in \Omega, T(z(\omega))=z(\omega)$. Hence $S(z(\omega))=T(z(\omega))=z(\omega)$. This shows that $z(\omega)$ is a common random fixed point of $S$ and $T$.

\section{Uniqueness}

Let $v(\omega)$ be another random fixed point common to $S$ and $T$, that is, for $\omega \in \Omega, S(v(\omega))=T(v(\omega))=v(\omega)$. Then for $\omega \in \Omega$, we have

$$
\begin{aligned}
d(z(\omega), v(\omega))= & d(S(z(\omega)), T(v(\omega))) \\
\leq & h \max \{d(z(\omega), v(\omega)), \\
& \frac{d(z(\omega), S(z(\omega)))+d(v(\omega), T(v(\omega)))}{2}, \\
& \left.\frac{d(z(\omega), T(v(\omega)))+d(v(\omega), S(z(\omega)))}{2}\right\} \\
= & h \max \{d(z(\omega), v(\omega)), 0, d(z(\omega), v(\omega))\} \\
\leq & h d(z(\omega), v(\omega)) \\
< & d(z(\omega), v(\omega)), \text { since } 0<h<1,
\end{aligned}
$$

a contradiction. Hence $z(\omega)=v(\omega)$ and so $z(\omega)$ is a unique common random fixed point of $S$ and $T$. This completes the proof.

Corollary 3.2. Let $(X, d)$ be a complete cone random metric space with respect to a cone $P$ and let $M$ be a nonempty separable closed subset of $X$. Let $T$ be a continuous random operator defined on $M$ such that for $\omega \in \Omega$, $T(\omega,):. \Omega \times M \rightarrow M$ satisfying the condition

$$
\begin{aligned}
d(T(x(\omega)), T(y(\omega))) \leq & h \max \{d(x(\omega), y(\omega)), \\
& \frac{d(x(\omega), T(x(\omega)))+d(y(\omega), T(y(\omega)))}{2}, \\
& \left.\frac{d(x(\omega), T(y(\omega)))+d(y(\omega), T(x(\omega)))}{2}\right\},
\end{aligned}
$$

for all $x, y \in X ; 0<h<1$ and $\omega \in \Omega$. Then $T$ has a unique random fixed point in $X$. 
Proof. The proof of the Corollary 3.2 immediately follows by taking $S=T$ in Theorem 3.1. This completes the proof.

Since each of the conditions $\left(z_{1}\right)-\left(z_{3}\right)$ included in (2.4). Hence from Theorem 3.1, we obtain the following results as corollaries by taking $S=T$.

Corollary 3.3. Let $(X, d)$ be a complete cone random metric space with respect to a cone $P$ and let $M$ be a nonempty separable closed subset of $X$. Let $T$ be a random operator defined on $M$ such that for $\omega \in \Omega, T(\omega,):. \Omega \times M \rightarrow$ $M$ satisfying the condition

$$
d(T(x(\omega)), T(y(\omega))) \leq a d(x(\omega), y(\omega)),
$$

for all $x, y \in X, a \in(0,1)$ and $\omega \in \Omega$. Then $T$ has a unique random fixed point in $X$.

Corollary 3.4. ([19], Corollary 3.2) Let $(X, d)$ be a complete cone random metric space with respect to a cone $P$ and let $M$ be a nonempty separable closed subset of $X$. Let $T$ be a continuous random operator defined on $M$ such that for $\omega \in \Omega, T(\omega,):. \Omega \times M \rightarrow M$ satisfying the condition

$$
d(T(x(\omega)), T(y(\omega))) \leq b[d(x(\omega), T(x(\omega)))+d(y(\omega), T(y(\omega)))]
$$

for all $x, y \in X, b \in\left(0, \frac{1}{2}\right)$ and $\omega \in \Omega$. Then $T$ has a unique random fixed point in $X$.

Corollary 3.5. (19], Corollary 3.3) Let $(X, d)$ be a complete cone random metric space with respect to a cone $P$ and let $M$ be a nonempty separable closed subset of $X$. Let $T$ be a continuous random operator defined on $M$ such that for $\omega \in \Omega, T(\omega,):. \Omega \times M \rightarrow M$ satisfying the condition

$$
d(T(x(\omega)), T(y(\omega))) \leq c[d(x(\omega), T(y(\omega)))+d(y(\omega), T(x(\omega)))]
$$

for all $x, y \in X, c \in\left(0, \frac{1}{2}\right)$ and $\omega \in \Omega$. Then $T$ has a unique random fixed point in $X$.

Corollary 3.6. Let $(X, d)$ be a complete cone random metric space with respect to a cone $P$ and let $M$ be a nonempty separable closed subset of $X$. Let $T$ be a continuous random operator defined on $M$ such that for $\omega \in \Omega$, $T(\omega,):. \Omega \times M \rightarrow M$ satisfying a $Z$-operator. Then $T$ has a unique random fixed point in $X$.

Remark 3.1. Theorem 3.1 extends Theorem $Z$ of [30] to the case of more general contractive condition and from complete metric space to cone random metric space. 
Remark 3.2. Our results also extend and generalize the corresponding results of Mehta et al. [19] (Bull. Cal. Math. Soc. 103(4) (2011), 303-310) and some others from the current existing literature.

Example 3.1. Let $M=R$ and $P=\{x \in M: x \geq 0\}$, also $\Omega=[0,1]$ and $\Sigma$ be the sigma algebra of Lebesgue's measurable subset of $[0,1]$. Let $X=$ $[0, \infty)$ and define a mapping $d:(\Omega \times X) \times(\Omega \times X) \rightarrow M$ by $d(x(\omega), y(\omega))=$ $|x(\omega)-y(\omega)|$. Then $(X, d)$ is a cone random metric space. Define random operator $T$ form $(\Omega \times X)$ to $X$ as $T(\omega, x)=\frac{1-\omega^{2}+2 x}{3}$. Also sequence of mapping $\xi_{n}: \Omega \rightarrow X$ is defined by $\xi_{n}(\omega)=\left(1-\omega^{2}\right)^{1+(1 / n)}$ for every $\omega \in \Omega$ and $n \in N$. Define measurable mapping $\xi: \Omega \rightarrow X$ as $\xi(\omega)=\left(1-\omega^{2}\right)$ for every $\omega \in \Omega$. Hence $\left(1-\omega^{2}\right)$ is the random fixed point of the random operator $T$.

Example 3.2. Let $M=R$ and $P=\{x \in M: x \geq 0\}$, also $\Omega=[0,1]$ and $\Sigma$ be the sigma algebra of Lebesgue's measurable subset of $[0,1]$. Let $X=$ $[0, \infty)$ and define a mapping $d:(\Omega \times X) \times(\Omega \times X) \rightarrow M$ by $d(x(\omega), y(\omega))=$ $|x(\omega)-y(\omega)|$. Then $(X, d)$ is a cone random metric space. Define random operators $S$ and $T$ form $(\Omega \times X)$ to $X$ as $S(\omega, x)=\frac{1-\omega^{2}+x}{2}$ and $T(\omega, x)=$ $\frac{1-\omega^{2}+2 x}{3}$. Also sequence of mapping $\xi_{n}: \Omega \rightarrow X$ is defined by $\xi_{n}(\omega)=(1-$ $\left.\omega^{2}\right)^{3+(1 / n)}$ for every $\omega \in \Omega$ and $n \in N$. Define measurable mapping $\xi: \Omega \rightarrow X$ as $\xi(\omega)=\left(1-\omega^{2}\right)$ for every $\omega \in \Omega$. Hence $\left(1-\omega^{2}\right)$ is a common random fixed point of the random operators $S$ and $T$.

\section{Acknowledgement}

The author would like to thanks the anonymous referee for his careful reading and valuable suggestions to improve the quality of the paper.

\section{References}

[1] M. Abbas and G. Jungck, Common fixed point results for non commuting mappings without continuity in cone metric spaces, J. Math. Anal. Appl., 341, (2008), 416-420

[2] M. Arshad and P. Vetro A. Azam, On Edelstein type multivalued random operators, Hacet. J. Math. Stat., 42(3), (2013), 223-229

[3] M. Arshad and A. Shoaib, Fixed points of multivalued mappings in fuzzy metric spaces, Proceedings of the world congress on engineering, July 4-6, (2012), p.664-664 
[4] I. Beg, Random fixed points of random operators satisfying semicontractivity conditions, Mathematics Japonica, 46(1), (1997), 151-155

[5] I. Beg, Approximation of random fixed points in normed spaces, Nonlinear Anal., 51(8), (2002), 1363-1372

[6] I. Beg and M. Abbas, Equivalence and stability of random fixed point iterative procedures, J. Appl. Math. Stoch. Anal., (2006), (2006), 19

[7] I. Beg and M. Abbas, Iterative procedures for solutions of random operator equations in Banach spaces, J. Math. Anal. Appl., 315(1), (2006), 181-201

[8] A.T. Bharucha-Reid, Random Integral equations Mathematics in Science and Engineering, J. Math. Anal. Appl., 96, (1972), .

[9] A.T. Bharucha-Reid, Fixed point theorems in Probabilistic analysis, Bull. Amer. Math. Soc., 82(5), (1976), 641-657

[10] L.B. Ciric, A generalization of Banach principle, Proc. Amer. Math. Soc., 45, (1974), $727-730$

[11] S.K. Chatterjee, Fixed point theorems compactes, Rend. Acad. Bulgare Sci., 25, (1972), 727-730

[12] O. Hanš, Reduzierende zufallige transformationen, Czech. Math. J., 7(82), (1957), $154-158$

[13] O. Hanš, Random operator equations, Proceeding of the 4th Berkeley Symposium on Mathematical Statistics and Probability, University of California Press, California, II, (1961), 185-202

[14] C.J. Himmelberg, Measurable relations, Fund. Math., 87, (1975), 53-72

[15] L.-G. Huang and X. Zhang, Cone metric spaces and fixed point theorems of contractive mappings, J. Math. Anal. Appl., 332(2), (2007), 1468-1476

[16] D. Ilic and V. Rakocevic, Common fixed points for maps on cone metric space, J. Math. Anal. Appl., 341, (2008), 876-882

[17] S. Itoh, Random fixed point theorems with an application to random differential equations in Banach spaces, J. Math. Anal. Appl., 67(2), (1979), 261-273

[18] R. Kannan, Some results on fixed point theorems, Bull. Cal. Math. Soc., 60, (1969), $71-78$

[19] A.D. Singh and Vanita Ben Dhagat Smriti Mehta, Fixed point theorems for weak contraction in cone random metric spaces, Bull. Cal. Math. Soc., 103(4), (2011), $303-310$

[20] N.S. Papageorgiou, Random fixed point theorems for measurable multifunctions in Banach spaces, Proc. Amer. Math. Soc., 97(3), (1986), 507-514

[21] N.S. Papageorgiou, On measurable multifunctions with stochastic domain, J. Austra. Math. Soc. Series A, 45(2), (1988), 204-216

[22] R. Penaloza, A characterization of renegotiation proof contracts via random fixed points in Banach spaces, working paper 269, Department of Economics, University of Brasilia, Brasilia, December, (2002) 
Vol. LIII (2015) Random fixed point theorems for Ciric quasi ......

[23] A. Petrusel, Generalized multivalued contractions, Nonlinear Anal. (TMA), 47(1), (2001), 649-659

[24] G. Petrusel and A. Petrusel, Multivalued contractions of Feng-Liu type in complete gauge spaces, Carpth. J. Math. Anal. Appl., 24, (2008), 392-396

[25] Sh. Rezapour and R. Hamlbarani, Some notes on the paper "Cone metric spaces and fixed point theorems of contractive mappings", J. Math. Anal. Appl., 345(2), (2008), 719-724

[26] V.M. Sehgal and S.P. Singh, On random approximations and a random fixed point theorem for set valued mappings, Proc. Amer. Math. Soc., 95(1), (1985), 91-94

[27] P. Vetro, Common fixed points in cone metric spaces, Rend. Circ. Mat. Palermo (2), 56(3), (2007), 464-468

[28] D.H. Wagner, Survey of measurable selection theorem, SIAM J. Control Optim., 15(5), (1977), 859-903

[29] P.P. Zabrejko, $K$-metric and $K$-normed linear spaces: survey, Collec. Math., 48(46), (1997), 825-859

[30] T. Zamfirescu, Fixed point theorems in metric space, Arch. Math. (Basel), 23, (1972), 292-298

G. S. Saluja

Department of Mathematics,

Govt. Nagarjuna P.G. College of Science,

Raipur - 492010 (C.G.), India.

E-mail: saluja1963@gmail.com

Received: 20.07.2013

Accepted: 11.03.2015 